\title{
Improving Evaluation of Primary Gastric Malignancies by Distending the Stomach with Milk Immediately Before ${ }^{18}$ F-FDG PET Scanning
}

\author{
Zhaohui Zhu ${ }^{1}$, Fang $\mathrm{Li}^{1}$, Yilei $\mathrm{Mao}^{2}$, Wuying Cheng ${ }^{1}$, Xin Cheng ${ }^{1}$, and Yonghong Dang ${ }^{1}$ \\ ${ }^{I}$ Department of Nuclear Medicine, Peking Union Medical College Hospital, Chinese Academy of Medical Sciences and Peking Union \\ Medical College, Beijing, China; and ${ }^{2}$ Department of Surgery, Peking Union Medical College Hospital, Chinese Academy of Medical \\ Sciences and Peking Union Medical College, Beijing, China
}

\begin{abstract}
This study was designed to investigate the feasibility and effectiveness of a modified protocol for ${ }^{18} \mathrm{~F}-\mathrm{FDG}$ PET that was proposed to improve the identification of primary gastric malignancies. Methods: In the modified protocol, patients were asked to drink $300-500 \mathrm{~mL}$ of cow milk to distend the stomach immediately before PET scans instead of fasting all along. For investigation of the influence of ingested milk on ${ }^{18} \mathrm{~F}-\mathrm{FDG}$ distributions, 43 nondiabetic patients without documented gastric diseases underwent both empty- and distended-stomach PET scans (79 and 72 scans, respectively) in their serial follow-up studies. For the evaluation of proven gastric malignancies, 24 patients who underwent distended-stomach PET scans were compared with 17 patients who underwent conventional empty-stomach examinations. Results: Ingestion of milk nearly $1 \mathrm{~h}$ after ${ }^{18} \mathrm{~F}-\mathrm{FDG}$ injection had no significant influence on distributions to the heart $(P=0.16)$, mediastinum $(P=0.50)$, and liver $(P=0.49)$, whereas the percentages of intense and moderate uptake in the stomach changed from $38.0 \%$ and $59.5 \%$ to $0 \%$ and $11.1 \%$, respectively. With the normal gastric wall distended, malignant lesions were observed with higher contrast and clearer outlines, and some of them were detected at a small size $(1.2 \mathrm{~cm})$ at an early stage and with mild uptake. Conclusion: Gastric distention with milk just before ${ }^{18} \mathrm{~F}-\mathrm{FDG}$ PET is a simple and effective method for improving the evaluation of primary gastric malignancies.
\end{abstract}

Key Words: PET; ${ }^{18}$ F-FDG; gastric malignancy; physiologic uptake; gastric distention

J Nucl Med Technol 2008; 36:25-29

DOI: 10.2967/jnmt.107.044081

$\mathbf{G}$

astric cancer is one of the most common malignancies, especially in Asian populations. It has been listed as the second leading cause of cancer-related death worldwide (1). Early detection and subsequent curative surgical removal provide the only chances for long-time survival (2). Although

Received Jun. 10, 2007; revision accepted Dec. 26, 2007.

For correspondence or reprints contact: Zhaohui Zhu, MD, Department of Nuclear Medicine, Peking Union Medical College Hospital, No.

1 Shuaifuyuan, Wangfujing St., Dongcheng District, Beijing 100730, China.

E-mail: zzh_1969@yahoo.com.cn or zhuzhh@pumch.cn

COPYRIGHT ( 2008 by the Society of Nuclear Medicine, Inc.
PET with ${ }^{18} \mathrm{~F}-\mathrm{FDG}$ as the tracer has been extensively used in oncology, it has limited value in the evaluation of primary gastric malignancies when routinely performed under fasting status (3), mainly because of the high level of physiologic uptake in the contracted stomach (4-7).

To improve the evaluation of primary gastric malignancies, we proposed a modified PET protocol to distend the stomach immediately before ${ }^{18}$ F-FDG PET and investigated its feasibility and effectiveness in clinical studies.

\section{MATERIALS AND METHODS}

\section{Patients}

Two groups of patients were involved in the study: one was selected to prove the feasibility of the modified protocol, and the other was selected to investigate its effectiveness in evaluating primary gastric malignancies. All of the patients were referred to the PET center at Peking Union Medical College Hospital for clinical purposes. The diagnoses of the patients are shown in Table 1.

Group 1 included 43 patients (18 women and 25 men; $57 \pm 12$ [mean \pm SD] y). They were selected to analyze the influence of milk ingestion on ${ }^{18} \mathrm{~F}-\mathrm{FDG}$ distributions in the body. All patients underwent both empty-stomach PET scans (79 scans, 1-6 scans per patient) and milk-distended-stomach PET scans (72 scans, 1-7 scans per patient) in their serial follow-up PET studies of nongastric malignancies; those with documented gastric diseases or diabetes mellitus were excluded. The intervals for paired PET scans under both conditions (empty stomach and distended stomach) ranged from 2 to 64 mo $(24 \pm 15$ mo [mean $\pm \mathrm{SD}])$.

Group 2 contained 41 patients with proven gastric malignancies. Twenty-four of the patients ( 8 women and 16 men; $60 \pm 14 \mathrm{y}$ ) underwent distended-stomach PET studies to evaluate primary tumors, and the other 17 patients ( 3 women and 14 men; $62 \pm 16 \mathrm{y}$ ) underwent routine empty-stomach PET scans. The PET findings were compared with those of gastroscopy or surgery for each patient.

\section{PET Protocol}

The patients fasted for at least $4 \mathrm{~h}$. The blood glucose level was tested for each patient; this level should be less than $6.7 \mathrm{mmol} / \mathrm{L}$ before intravenous injection of ${ }^{18} \mathrm{~F}-\mathrm{FDG}(7.4 \mathrm{MBq} / \mathrm{kg})$. The patients then rested quietly in a warm and dark room for $1 \mathrm{~h}$ for the 
TABLE 1

Groups and Diagnoses of Patients

\begin{tabular}{lc}
\hline \multicolumn{1}{c}{ Groups and diagnoses } & No. of patients \\
\hline Group 1: serial studies under both & 43 \\
conditions (distended stomach $[n=72]$ & \\
and empty stomach $[n=79]$ ) & 18 \\
Lung cancer & 6 \\
Non-Hodgkin's lymphoma & 6 \\
Ovarian cancer & 2 \\
Breast cancer & 2 \\
Cervical cancer of uterus & 9 \\
Other & \\
Group 2: gastric malignancies & 24 \\
Underwent distended-stomach PET study & 15 \\
Adenocarcinoma, not mucinous & 1 \\
Adenocarcinoma, mucinous & 5 \\
Signet-ring cell carcinoma & 3 \\
Non-Hodgkin's lymphoma & 17 \\
Underwent empty-stomach PET study & 11 \\
Adenocarcinoma, not mucinous & 2 \\
Adenocarcinoma, mucinous & 3 \\
Signet-ring cell carcinoma & 1 \\
Non-Hodgkin's lymphoma &
\end{tabular}

distribution of ${ }^{18} \mathrm{~F}$-FDG. The selected patients, who had no history of milk allergy, were asked to try to drink $300-500 \mathrm{~mL}$ of pure milk within $5 \mathrm{~min}$ before PET; the same procedures were used for routine empty-stomach PET, except for this step. All of the scans were performed with an ECAT EXACT HR + PET system (Siemens/ CTI). The emission and transmission scans were acquired intermittently for 6 and $3 \mathrm{~min}$, respectively, per bed position in a 2dimensional mode. From the floor of the pelvis to the chin, each scan included 5 or 6 bed positions and lasted for nearly $1 \mathrm{~h}$. The acquisition of the stomach region was usually obtained 20-30 min after the ingestion of milk. The images were reconstructed by use of an iterative algorithm (ordered-subsets expectation maximization) with attenuation correction and were displayed in coronal, axial, and sagittal views as well as in cinematic views of maximum intensity projections.

\section{Data Analysis}

Three experienced nuclear medicine physicians interpreted the PET images on a high-resolution computer screen. In visual analysis, gastric uptake was designated as intense, moderate, or mild (or indistinguishable) uptake, corresponding to uptake that was remarkably higher than, almost equal to, or remarkably lower than that in the liver, respectively. The shape and extent of any focal uptake and its relationship to the stomach were noted. For semiquantitative analysis, a region of interest was drawn over the corresponding region, and the average standardized uptake value (SUV) corrected for total body weight was calculated.

\section{Statistical Analysis}

SSPS software (version 12.0 for Windows) was used for statistical analysis. To determine the differences in the SUVs for the gastric wall, heart, mediastinum, and liver between the distendedstomach and the empty-stomach PET examinations for the same patients, we used the general linear model for repeated measures to analyze paired studies from the same patients. If more than one scan was obtained under each condition, then adjacent scans obtained under the 2 conditions were selected. A $P$ value of $<0.05$ was considered statistically significant.

\section{RESULTS}

\section{Influence of Milk Ingestion on ${ }^{18}$ F-FDG Distributions}

Drinking 300-500 mL of milk immediately before PET effectively distended the stomach in most patients, and the level of physiologic gastric uptake was remarkably decreased through gastric distention (Fig. 1 and Table 2). In the 43 patients (group 1) who underwent serial PET examinations under both conditions, the percentages of intense, moderate, and mild uptake levels in the stomach changed from $38.0 \%, 59.5 \%$, and $2.5 \%$ in the empty-stomach condition ( 79 scans) to $0 \%, 11.1 \%$, and $89.9 \%$, respectively, in the distended-stomach condition (72 scans).

In the semiquantitative analysis, the adjacent empty- and distended-stomach PET scans of the 43 patients with serial PET follow-up examinations were compared to investigate the influence of milk ingestion on ${ }^{18} \mathrm{~F}$-FDG distributions in different organs and tissues. The interval between the paired PET examinations was $24 \pm 15$ mo (mean $\pm \mathrm{SD} ; n=43$ ). Statistical analysis showed that the SUVs in the gastric wall were significantly decreased after gastric distention (Table 2). However, the SUVs in the heart, mediastinum, and liver, which were most likely to change with the increase in the blood glucose level after food ingestion, were not significantly different on the paired PET images obtained from the same patient.

\section{Evaluation of Gastric Malignancies}

Of the patients with proven gastric malignancies (group 2), 24 underwent distended-stomach PET studies. With the stomach distended, gastric lesions were observed with high contrast and clear outlines, and the distended normal gastric wall had only mild or indistinguishable uptake (Fig. 2). The filled gastric cavity without radioactivity also increased contrast. Therefore, the gastric malignancies were diagnosed with more confidence, and some of them were detected at a small size (up to $1.2 \mathrm{~cm}$ ) and with mild uptake (up to an SUV of 1.76). The sensitivity in this group of patients was $95.8 \%$ (23/24). The only patient with negative findings had a mucinous adenocarcinoma that had an SUV of 1.32 at the corresponding region and that was indistinguishable from the moderately distended gastric wall (SUVs=0.84-1.61).

Seventeen patients in group 2 underwent conventional empty-stomach PET scans. Twelve of them showed moderate to intense uptake in the gastric wall that was not related to the malignancies revealed by endoscopy or surgery (Fig. 2). Among these patients, 4 underwent spot imaging of the stomach after gastric distention with food, which remarkably decreased the uptake in the normal gastric wall and accurately defined the range of malignant lesions. In 5 patients with only mild uptake in the normal gastric wall, the stomach had actually been moderately distended by remnant food, 


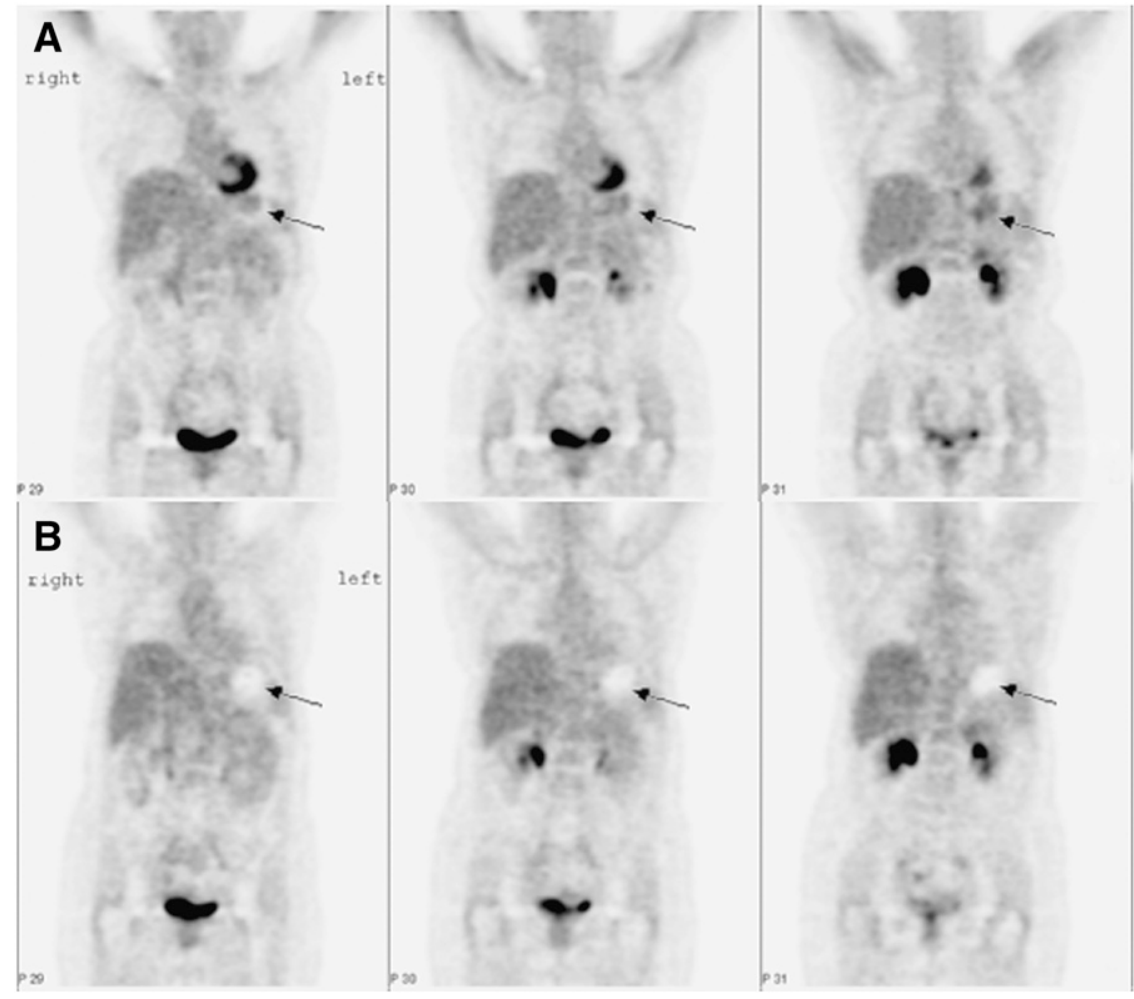

FIGURE 1. Comparison of emptystomach and distended-stomach PET studies from same patient. Patient $(F$, 57 y) underwent serial follow-up of cervical carcinoma of uterus after curative surgical removal and radiotherapy. Interval between 2 studies was 7 mo. Stomach (arrow) showing moderate to intense ${ }^{18} \mathrm{~F}$ FDG uptake in empty-stomach study (A, coronal images) changed to "hollow" region in distended-stomach study ( $B$, coronal images). Note that heart, with intense uptake under empty-stomach condition, did not show prominent uptake after gastric distention with milk. possibly because of delayed gastric emptying caused by the gastric malignancies.

\section{DISCUSSION}

High-level physiologic uptake in the stomach is commonly observed on ${ }^{18} \mathrm{~F}-\mathrm{FDG}$ PET and PET/CT images under conventional fasting status. In the present study, the percentages of intense and moderate uptake in the stomach reached $38.0 \%$ and $59.5 \%$, respectively, in the 79 PET scans obtained under the empty-stomach condition. In another report including $300{ }^{18} \mathrm{~F}$-FDG PET studies in a healthy Chinese population, over $55 \%$ of the patients had gastric uptake with SUVs of greater than 2.5, and only $15 \%$ had indistinguishable uptake (4). In a report from The Netherlands, de Groot et al. reported that for 175 nondiabetic patients, $46 \%$ had clear stomach uptake and 5\% had high stomach uptake (5). Therefore, intense ${ }^{18} \mathrm{~F}-\mathrm{FDG}$ uptake in the stomach has been found to be common in both Eastern and Western populations despite their dietary differences.
Nonspecific uptake in the stomach may be related to its richness in smooth muscle and digestive glands, and the pattern of physiologic gastric uptake has been described in other reports $(6,7)$. When the stomach is full, the normal gastric wall will be remarkably distended and the level of uptake will seem relatively lower, whereas malignant lesions will not show much change in appearance because of their low elasticity; therefore, gastric distention may be one of the best ways to increase tumor-to-nontumor contrast.

Tian et al. conducted a prospective study to evaluate a vesicant-modified ${ }^{18} \mathrm{~F}$-FDG PET technique for the diagnosis and staging of gastric malignancies (8). When the imaging field was moved upward to the umbilical level, 2-3 g of vesicant powder (producing $120 \mathrm{~mL}$ of $\mathrm{CO}_{2}$ gas per gram within minutes after dissolving) was given orally and swallowed with $40-60 \mathrm{~mL}$ of water. This procedure improved the diagnostic confidence in their experience. Yun et al. used the water ingestion method to eliminate physiologic ${ }^{18} \mathrm{~F}$-FDG uptake in the contracted remnant stomach after subtotal gastrectomy (9). After whole-body

TABLE 2

SUVs in Gastric Wall, Heart, Mediastinum, and Liver in Same Patient According to Scan Condition

\begin{tabular}{lcrr}
\hline & \multicolumn{3}{c}{ Mean \pm SD SUV in: } \\
\cline { 2 - 4 } Organ or tissue & Stomach distended with milk $(n=43)$ & $F$ & 184.47 \\
\hline Gastric wall & $2.21 \pm 0.58$ & $0.93 \pm 0.32$ & 0.00 \\
Heart & $3.13 \pm 1.85$ & $2.71 \pm 1.54$ & 0.16 \\
Mediastinum & $1.38 \pm 0.23$ & $1.36 \pm 0.22$ & 0.00 \\
Liver & $1.85 \pm 0.30$ & $1.82 \pm 0.29$ & 0.47 \\
\hline
\end{tabular}




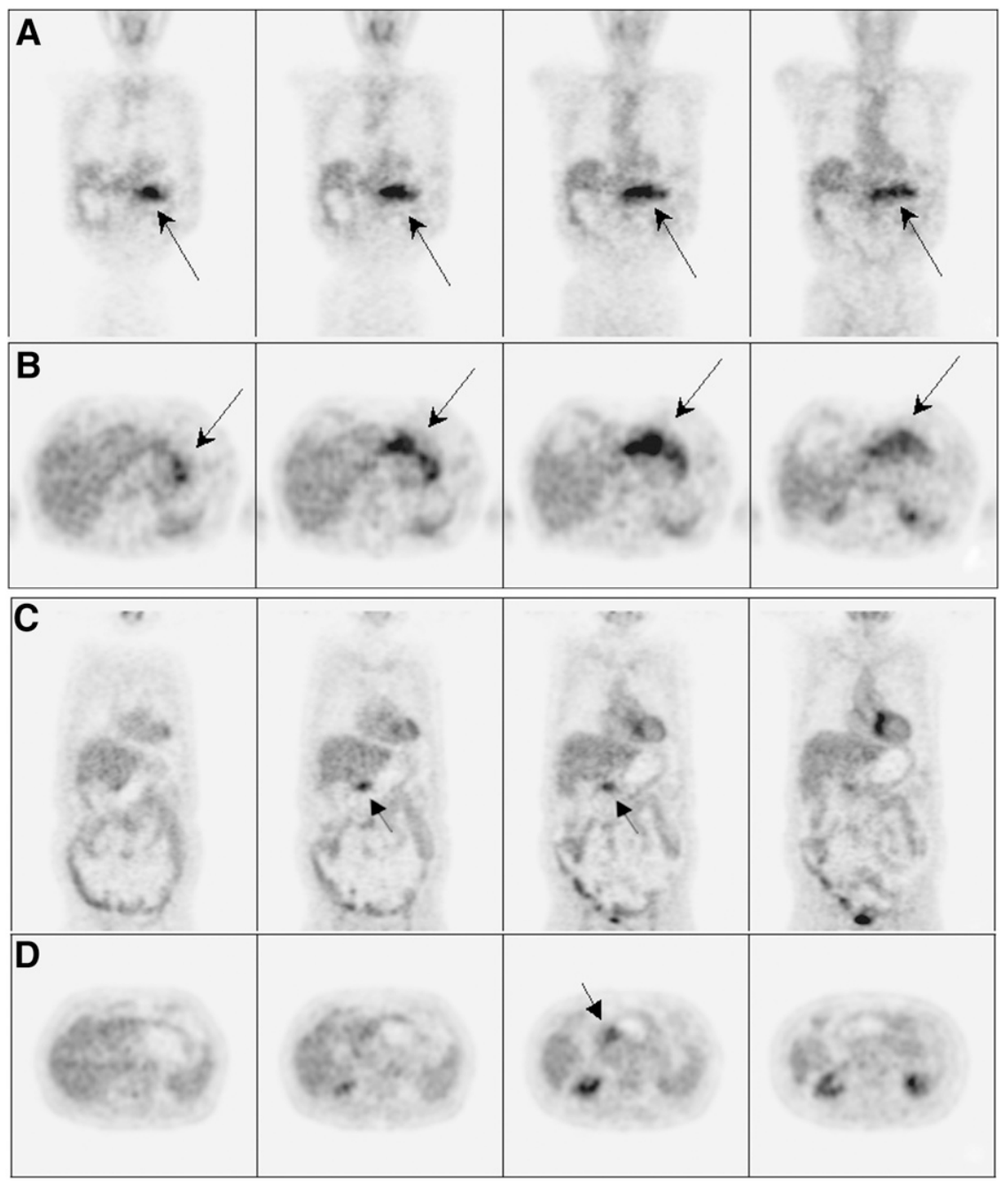

FIGURE 2. Comparison of emptystomach and distended-stomach PET scans in 2 patients with moderately to poorly differentiated adenocarcinomas at antrum of stomach. First patient (M, $90 \mathrm{y})$ underwent empty-stomach PET scan. Coronal (a) and transaxial (b) images showed intense uptake in whole stomach (arrow), which obscured lesion located at antrum. Second patient (M, 62 y) underwent distended-stomach PET scan. On coronal (c) and transaxial (d) images, lesion (arrow) was clearly outlined, and normal gastric wall was distended.

imaging, patients were asked to drink water, and then spot imaging of the stomach was performed. This simple procedure also improved the diagnostic accuracy of ${ }^{18}$ F-FDG PET in patients with suspected recurrence of carcinoma in the remnant stomach. However, both water and gas cannot remain long in the stomach; therefore, a PET scan must be completed as soon as possible after gastric distention. In a previous study, we used milk and bread to distend the stomach after conventional whole-body imaging and then performed stomach spot imaging; this technique also improved the evaluation of suspected primary gastric tumors (10).

In the present study, we used a modified PET protocol in which patients were asked to drink cow milk to distend the stomach immediately before whole-body scanning. Two aspects of gastric distention with food before scanning should be considered. The first aspect is feasibility. The food used must be easy to ingest within a few minutes and, more importantly, the ingested food should not affect the distribution of ${ }^{18} \mathrm{~F}-\mathrm{FDG}$ among other organs. If the food is ingested $1 \mathrm{~h}$ after injection and the acquisition can be finished not long after ingestion, the potential effect of hyperglycemia on the distribution of ${ }^{18} \mathrm{~F}-\mathrm{FDG}$ may be small. In the present study, we confirmed this hypothesis by examining 43 patients with serial PET scans under 2 conditions (empty stomach and distended stomach); we found no significant differences between the 2 conditions for the same patients with regard to the distribution of ${ }^{18} \mathrm{~F}-\mathrm{FDG}$ in the heart, mediastinum (blood pool), and liver, which were most likely to be influenced by the food ingestion. In some cases, intense cardiac uptake could be observed in the empty-stomach condition, but it was not prominent after gastric distention with milk (Fig. 1); this result indicated that milk ingestion did not cause the heart to use glucose during scanning.

The second aspect is efficiency. Drinking $300-500 \mathrm{~mL}$ of milk just before the PET scans effectively distended most of the stomachs in the present study; the percentages of intense and moderate gastric uptake changed from $38.0 \%$ and $59.5 \%$ in the empty-stomach condition to $0 \%$ and $11.1 \%$, respectively, after gastric distention. The average SUVs in the gastric wall significantly decreased, from $2.21 \pm 0.58$ to $0.93 \pm 0.32$ (mean $\pm \mathrm{SD}, n=43 ; P<0.001$ ), even if the acquisition of the stomach region took place nearly $20-30$ 
min after the ingestion of milk. Furthermore, by raising the tumor-to-nontumor contrast through distending the normal gastric wall and filling the stomach with food (nonradioactive), this simple procedure remarkably improved the evaluation of gastric malignancies (Fig. 2).

However, gastric distention with cow milk also had its limitations in the present study. First, some patients were allergic to cow milk or felt uncomfortable after drinking milk. For these patients, we recommended drinking water as an alternative, but the water was nearly emptied when the acquisition of the stomach region took place 20-30 min later. We next tried soya-bean juice instead; it had an effect similar to that of milk ingestion. Second, the stomachs were not fully distended in some patients. In future studies, we will try to add some solid food (e.g., bread and egg) to further delay the stomach-emptying process. In any event, a PET scan lasting for nearly $1 \mathrm{~h}$ from the pelvis to the neck is too slow for current practice. Many new PET systems can finish scanning within $20 \mathrm{~min}$; therefore, this problem will be less prominent in the future.

\section{CONCLUSION}

Drinking $300-500 \mathrm{~mL}$ of cow milk immediately before PET can effectively distend the stomach without significant influence on ${ }^{18} \mathrm{~F}-\mathrm{FDG}$ distributions and improve the evaluation of gastric malignancies by increasing the contrast be- tween primary gastric tumors and the normal gastric wall. We suggest that this simple modification of the PET protocol be used routinely for ${ }^{18} \mathrm{~F}$-FDG PET scans, especially in populations with a high incidence of gastric malignancies.

\section{REFERENCES}

1. Crew KD, Neugut AI. Epidemiology of gastric cancer. World J Gastroenterol. 2006; 12:354-362.

2. Jansen EP, Boot H, Verheij M, et al. Optimal locoregional treatment in gastric cancer. J Clin Oncol. 2005;23:4509-4517.

3. Mochiki E, Kuwano H, Katoh H, et al. Evaluation of ${ }^{18} \mathrm{~F}$-2-deoxy-2-fluoro-Dglucose positron emission tomography for gastric cancer. World J Surg. 2004; 28:247-253.

4. Ding Y, Zhang SW, Tian JH, et al. The diagnosis of gastric cancer with ${ }^{18} \mathrm{~F}-\mathrm{FDG}$ PET and its influencing factors. Chin J Med Imaging. 2003;11:320-323.

5. de Groot M, Meeuwis APW, Kok PJM, et al. Influence of blood glucose level, age and fasting period on non-pathological FDG uptake in heart and gut. Eur $J$ Nucl Med Mol Imaging. 2005;32:98-101.

6. Salaun PY, Grewal RK, Dodamane I, et al. An analysis of the ${ }^{18}$ F-FDG uptake pattern in the stomach. $J$ Nucl Med. 2005;46:48-51.

7. Koga H, Sasaki M, Kuwabara Y, et al. An analysis of the physiological FDG uptake pattern in the stomach. Ann Nucl Med. 2003;17:733-738.

8. Tian J, Chen L, Wei B, et al. The value of vesicant ${ }^{18} \mathrm{~F}$-fluorodeoxyglucose positron emission tomography $\left({ }^{18} \mathrm{~F}-\mathrm{FDG}\right.$ PET) in gastric malignancies. Nucl Med Commun. 2004;25:825-831.

9. Yun M, Choi HS, Yoo E, et al. The role of gastric distention in differentiating recurrent tumor from physiologic uptake in the remnant stomach on ${ }^{18} \mathrm{~F}-\mathrm{FDG}$ PET. J Nucl Med. 2005;46:953-957.

10. Zhu Z, Li F, Zhuang H. Gastric distention by ingesting food is useful in the evaluation of primary gastric cancer by FDG PET. Clin Nucl Med. 2007;32: 106-109. 